\title{
INFRARED LINE SHAPES IN ACTIVE GALACTIC NUCLEI
}

\author{
Rodger I. Thompson \\ Steward Observatory, University of Arizona
}

\begin{abstract}
This paper presents the complete spectrum of NGC 4151 from 0.87 to 2.5 $\mu \mathrm{m}$ as well as detail of the Paschen $\alpha$ profile in $3 \mathrm{C} 273$ at a resolution of about 5000 . Analysis of the several observed Fe II lines yields a most probable electron density of $10^{4}$ and a most likely temperature of $10^{4}$. The Fe II to $\mathrm{H}$ ratio is $2+/-1 \times 10^{-6}$ which is $5-7 \%$ of the available iron if the $\mathrm{Fe} / \mathrm{H}$ ratio is solar.
\end{abstract}

\section{Observations}

The complete 0.87 - $2.5 \mu \mathrm{m}$ spectrum of NGC 4151 was observed with the Grating Infrared Spectrometer (GRIS) at an average resolution of 5300. Only regions of severe telluric absorption are missing from the spectrum. This spectrum has the advantage of simultaneous and consistent observation of many spectral lines from both the narrow and broad line region plus overlap with the spectral region available to visible CCD spectrometers. The known variability of the continuum and broad line emission from NGC 4151 make the simultaneous observations very important.

\section{Results}

The major analysis is an examination of the Fe II lines using the new excitation parameters of Pradhan and Zhang (1993). Comparison of the solution of the equilibrium equations with the observed line ratios yields a most probable electron temperature of $10^{4} \mathrm{~K}$ and an electron density of $10^{4} \mathrm{~cm}^{-3}$. Some observations from Osterbrock et al 1993 were utilized in the analysis. The ratio of Fe II 1.644 $\mu \mathrm{m}$ to Paschen $\beta$ yields a FeII/H ratio of $2+/-1 \times 10^{-6}$ or about $6 \%$ of the iron for a solar $\mathrm{Fe} / \mathrm{H}$ ratio. This indicates significant grain evaporation or destruction in the Fe II emission region. This appears to be more consistent with fast shocks as the ionization mechanism as opposed to slow shocks. High energy photons could also play a role in destroying the grains. Not all of the line ratios are consistent with the determined density but they may suffer from blends or other observational effects.

The Paschen $\alpha$ emission in $3 \mathrm{C} 273$ is consistent with the line shapes in the optical region. There is no evidence in the present spectrum of previously reported $\mathrm{H}_{2}$ emission from the object.

\section{References}

Osterbrock, D.E., Shaw, R.A., and Veilleux, S. 1992, Ap.J., 352, 561.

Pradhan, A.K. and Zhang, H.L. 1993, ApJ (Letters), 409, L77. 